\title{
Le financement public des religions en Europe : statuts actuels et pratiques émergentes
}

Francis Messner

\section{(2) OpenEdition}

\section{Journals}

Édition électronique

URL : http://journals.openedition.org/rdr/1027

DOI : $10.4000 /$ rdr. 1027

ISSN : 2534-7462

Éditeur

Presses universitaires de Strasbourg

Édition imprimée

Date de publication : 10 mai 2016

Pagination : 23-37

ISBN : 978-2-86820-954-2

ISSN : 2493-8637

\section{Référence électronique}

Francis Messner, «Le financement public des religions en Europe : statuts actuels et pratiques

émergentes », Revue du droit des religions [En ligne], 1 | 2016, mis en ligne le 12 février 2020, consulté le 19 novembre 2020. URL : http://journals.openedition.org/rdr/1027 ; DOI : https://doi.org/10.4000/rdr. 1027

La revue du droit des religions est mise à disposition selon les termes de la Creative Commons Attribution - Pas d'Utilisation Commerciale 4.0 International - CC BY-NC 4.0. 


\section{LE FINANCEMENT PUBLIC DES RELIGIONS EN EUROPE : STATUTS ACTUELS ET PRATIQUES ÉMERGENTES}

\section{Francis MESSNER}

Université de Strasbourg / CNRS, Droit, Religion, Entreprise et Société (DRES)

\section{RÉSUMÉ}

Les divers régimes de financement public des cultes dans les pays européens sont aujourd'hui encore largement issus des évolutions historiques nées sous l'impulsion de la Réforme et des Lumières, et notamment des systèmes de compensation mis en place à la suite de la sécularisation des revenus des Églises. La légitimité de ces fondements tend cependant à perdre de sa pertinence dans le contexte économique et socioreligieux actuel, lequel suscite la mise en œuvre de nouveaux modes de soutien plus adaptés à la perception actuelle du rôle et de la diversité des confessions religieuses.

\section{ABSTRACT}

The various schemes of public funding of religions in European countries are rooted in History and more particularly the Reformation and the Enlightenment, including compensation schemes set up in exchange of secularization of religious assets. The legitimacy of these foundations tends to lose its relevance in the current economic and socio-religious context, which leads to the implementation of new modes of support more suited to the contemporary perception of the role played by religious groups and the growing religious diversity. 
T es modes actuels de financement direct ${ }^{1}$, privé et public, des cadres _religieux, des bâtiments, des activités cultuelles et des institutions religieuses sont en large partie issus du bénéfice ecclésiastique (beneficium), élément historique commun à l'Europe géographique. Le titulaire de l'office qui n'exerçait pas toujours la charge (sine cura), et dans ce cas la sous-traitait, percevait une rente, fruit d'un revenu constitué par la dîme et par un patrimoine immobilier. Le bénéfice a été considéré par l'Église catholique comme un instrument privilégié et quasi incontournable de financement, et cela jusqu'à une période récente ${ }^{2}$. Pour preuve, le concordat du 27 août 1953 entre le Saint-Siège et l'État espagnol envisageait dans son article 19 la création, par ailleurs irréaliste, d'un patrimoine ecclésiastique en vue d'assurer la dotation du culte et du clergé garantissant l'autonomie économique de l'Église catholique. De même, les canonistes français du XIX $X^{e}$ siècle avaient créé une fiction en consonance avec les revendications de l'Église définie comme une société parfaite. Ils considéraient que les curés et desservants concordataires (1802-1905) n'étaient pas des salariés stricto sensu, mais les titulaires d'un bénéfice constitué par le traitement versé par l'État, par le presbytère mis à la disposition par la commune ou la fabrique, les revenus de la mense curiale et le casuel dont la répartition entre le curé et l'établissement public du culte est fixée par les articles organiques du culte catholique. Le système de financement d'une religion par le biais de son patrimoine historique a peu ou prou été maintenu au Royaume-Uni, pour la seule Église d'Angleterre qui tire sa subsistance d'un fonds constitué de biens mobiliers et immobiliers ayant échappé à la sécularisation et des revenus d'une dotation versée par la couronne aux fins de compenser la suppression de la dîme au XIX e siècle. Le régime bénéficial qui ne correspondait plus aux exigences de la société moderne marquée par l'émergence du libéralisme a cependant pris fin dans de nombreux pays dès le $\mathrm{XVI}^{\mathrm{e}}$ siècle sous l'impulsion de la Réforme et des Lumières. Les biens de mainmorte constituant le bénéfice faisaient figure de frein au développement économique et la dîme était le plus souvent perçue sur les seuls produits agricoles, alors que se développait l'activité commerciale et industrielle. Ainsi en Suède, le patrimoine ecclésiastique a été dévolu à la

1. Le subventionnement des activités caritatives, éducatives ou sociales exercées sous la tutelle ou sous la responsabilité d'un organisme confessionnel n'entre pas dans le champ de cette présentation. Dans ce cas, il ne s'agit pas de l'exercice du culte, mais d'activités socio-éducatives subventionnées par les pouvoirs publics à l'instar d'associations non confessionnelles poursuivant les mêmes buts. - Sur la question du financement indirect, V. dans ce numéro, Curtit F., "La fiscalité des cultes en Europe: vers la fin de la singularité religieuse? ».

2. V. CIC 1917, canons 1409 à 1494. 
famille royale lors du passage du pays au luthéranisme $a u \mathrm{XVI}^{\mathrm{e}}$ siècle. Le roi s'était érigé en chef de l'Église à la place du pape et les évêques devenaient des fonctionnaires stipendiés. Au Danemark, les biens ont été répartis en trois parts égales, revenant respectivement à l'Église, au roi et au clergé3.

La sécularisation des biens ecclésiastiques a pris une forme plus radicale encore à la fin du XVIII ${ }^{\mathrm{e}}$ siècle en France et dans les territoires occupés à l'occasion de la Révolution. Les dîmes et les redevances qui en tiennent lieu sont supprimées par un arrêté de la Constituante des 4-11 août 1789 et quelques mois plus tard tous les biens ecclésiastiques sont mis à la disposition de la nation, conformément à l'article premier du décret du 2 novembre 1789 . Ces textes mettent fin à l'autonomie économique de l'Église catholique et simultanément à son quasi-monopole en matière d'instruction et de charité. La sécularisation des biens et sa contrepartie, la création d'un clergé salarié par l'État, ont généré la théorie dite de la « compensation » dont les prémices sont inscrites dans le décret du 2 novembre 1789 : les biens ecclésiastiques sont mis à la disposition de la nation, à la charge pour l'État de pourvoir de manière convenable aux frais de culte, à l'entretien de ses ministres et au soulagement des pauvres ${ }^{4}$. L'aliénation sans retour possible à l'Église des biens ecclésiastiques par les lois de la Révolution, par ailleurs entérinée par le Saint-Siège ${ }^{5}$, sera alors liée à l'instauration d'un budget étatique des cultes en substitution au système bénéficial, créé quelques mois plus tard par un décret du 14 avril 1790. Par conséquent, la Constitution civile du clergé du 12 juillet 1790 fixe le statut d'un clergé fonctionnarisé soumis à l'obligation de résidence et prévoit le versement d'un traitement aux prêtres occupant un des offices fixés par ce texte. Ce système fait long feu : un décret du 18 septembre 1794 préfigurant la séparation de 1905 dispose que la République française ne paie plus les frais ni les salaires d'aucun culte. Cette abstention de l'État en matière de financement a cependant été de courte durée. La convention de messidor (1801) et la loi du 18 germinal an X (1802) prévoient, dans le cadre de la réorganisation des cultes, un financement étatique des personnels cultuels calqué en grande partie sur la Constitution civile du clergé. L'obligation de rémunération fut à la suite de la Seconde Restauration inscrite dans les chartes constitutionnelles de 1814 (art. 7) et de 1830 (art. 6) ainsi que dans la constitution de 1848 (art. 8).

3. Pour la partie historique, V. Basdevant-Gaudemet B. et Messner F. (dir.), Les origines historiques du statut des confessions religieuses dans les pays de l'Union européenne, Paris, PUF, 1999.

4. Messner F., Le financement des Églises. Le système des cultes reconnus, 1801-1983, Strasbourg, Cerdic, 1984.

5. Art. 13 de la convention du 26 messidor an IX (1801). 
Les autres pays européens ont connu des évolutions analogues. En Allemagne, le processus initié par la Réforme et les Lumières débute dès 1795 et est confirmé par le recès impérial du 25 février 1803, alors qu'en Autriche l'étatisation des biens est intervenue dès 1780 sous l'impulsion du joséphisme. En Italie, le processus de réunification a accéléré la sécularisation des biens d'Église (1866-1867), déjà intervenue en 1837 pour la péninsule ibérique.

Si les Églises ont perdu leur souveraineté en matière économique, il n'est pas question pour autant de les priver de moyens d'existence. Les changements induits par la Réforme, les Lumières et le libéralisme économique du $\mathrm{XVI}^{\mathrm{e}}$ au $\mathrm{XIX}^{\mathrm{e}}$ siècle sont à l'origine des types de financement actuel des cultes (1) qui cèdent progressivement la place à de nouvelles modalités de subventionnement plus adaptées aux politiques religieuses contemporaines (2).

\section{LA DIVERSITÉ DES STATUTS ACTUELS}

Le financement des cultes dans les différents États européens est d'une grande diversité, chaque pays ayant conservé ses particularités. Il est cependant possible de retenir trois grandes catégories de modes de financement renvoyant à autant d'étapes historiques caractérisées par la survivance d'un avatar du système bénéficial (1.1), par le maintien d'un système de soutien par l'État (1.2) et enfin par une volonté de retrait plus ou moins abouti de la puissance publique (1.3).

\subsection{LES ÉGLISES VIVENT DE LEURS RENTES}

Un mode de financement adossé au maintien aménagé de la propriété ecclésiastique subsiste encore au Royaume-Uni pour la seule Église établie d'Angleterre, qui vit de ses rentes. Les biens mobiliers et immobiliers sont centralisés dans un fonds et gérés par une commission formée de représentants de l'Église établie et de membres de l'administration publique. Mais l'État ne subventionne ni l'Église établie, ni les Églises désétablies ou les autres confessions ${ }^{6}$ et n'intervient que dans le cadre du droit commun : entretien des monuments historiques affectés à l'exercice du culte ${ }^{7}$ et exonérations

6. McClean D., «State and Church in the United Kingdom», in Robbers G. (ed.), State and Church in the European Union, Baden Baden, Nomos , $2^{\text {nd }}$ ed. 2005, p. 553-575.

7. CRANMER F., « Living hand-to-mouth: regulating and funding religious heritage in the United Kingdom », in FORNEROD A. (ed.), Funding religious heritage, Farnham, Ashgate, 
fiscales des organisations sous statut de charities qui poursuivent des activités à caractère non lucratif au bénéfice de la collectivité, dont font partie les activités à but religieux. La Grèce participe d'une certaine manière de ce système. Les biens de l'Église orthodoxe ont été préservés alors que l'État prend en charge les rémunérations des ministres du culte, ce qui ne manque pas de susciter de virulentes critiques dans un pays fragilisé par une importante crise financière. Cette évolution paradoxale s'explique par le rôle important joué par la religion dominante en tant que facteur de conservation des valeurs traditionnelles et de l'identité des Hellènes. Le lien étroit entre la nation, le peuple, l'État et l'Église orthodoxe a déterminé le gouvernement grec à financer l'Église orthodoxe ${ }^{8}$. L'Église orthodoxe de Chypre a quant à elle transmis une partie de ses biens immobiliers à l'État qui verse un salaire aux prêtres orthodoxes des paroisses rurales en contrepartie de cette cession. Il rémunère également des prêtres d'autres Églises chrétiennes

\subsection{LES RELIGIONS SONT SOUTENUES PAR L'ÉTAT}

La deuxième catégorie de types de financement, de loin la plus importante, comprend les États qui prennent en charge la totalité ou une partie du financement des cultes ou mettent à disposition des confessions religieuses les services de l'administration fiscale. Les modes de soutien économique public se révèlent dans ce cas assez variés.

Suite à la sécularisation radicale des biens ecclésiastiques et à la suppression de la dîme en France et dans les territoires qu'elle occupait, les autorités publiques interviennent au titre du principe ou de la théorie de la compensation et prennent en charge l'ensemble du financement nécessaire au bon fonctionnement des cultes, en France jusqu'en 1905, en Belgique (anciennement département des Ardennes) et au Luxembourg (anciennement département des forêts). En Belgique par exemple, les cultes reconnus reçoivent aujourd'hui des aides publiques qui couvrent la totalité de leurs frais de fonctionnement : rémunération des ministres du culte et personnels assimilés et entretien des bâtiments affectés au « service public » des cultes et à la

2015, p. 53-74.

8. Papasthatis Ch. K., «State financial support in Greece », in Stati e confessioni religiose in Europa, modelli di finanziamento pubblico, scuola e fattore religioso $=$ Church and State in Europe. State financial support. Religion and the school, Milano, Giuffrè, 1992 ; « State and Church in Greece », in Robbers G. (ed.), op. cit., p. 115-138. 
laicité organisée ${ }^{9}$. Les chefs religieux, les ministres du culte et les personnels de secrétariat des autorités religieuses sont rétribués par l'État. Les établissements publics du culte local ou paroissial sont chargés de couvrir les dépenses de fonctionnement et d'investissement liées à l'exercice public du culte, qui comprennent les frais nécessaires à la célébration des cultes, les salaires et charges du personnel employé par l'établissement, les travaux d'embellissement, l'entretien, les réparations et la reconstruction des édifices du culte et des logements des ministres du culte. Les communes sont tenues de mettre un logement à disposition des ministres du culte nommés dans une circonscription paroissiale ou locale, ou à défaut de verser une indemnité de logement, et de prendre en charge les frais de culte en cas d'insuffisance des revenus des établissements publics du culte.

Dans d'autres États, un système de financement des cultes adossé à l'affiliation religieuse s'est développé à la suite de la Réforme, notamment dans les pays germaniques et scandinaves. En Allemagne, les sociétés religieuses (Religionsgesellchaft) peuvent collecter un impôt d'Église (Kirchensteuer) sur la base des rôles civils d'impôts et dans les conditions fixées par la législation de chaque État fédéré (Land). Cet impôt est versé par les personnes physiques qui sont imposables sur le revenu et qui appartiennent à une société religieuse constituée en collectivité de droit public, sous réserve que le nombre de ses membres soit suffisant. Les personnes qui souhaitent mettre fin à leur affiliation remplissent un formulaire de sortie auprès des pouvoirs publics locaux qui le transmettent à l'administration fiscale. Cet impôt cultuel, soit $80 \%$ du budget des sociétés religieuses concernées, représente 8 à $9 \%$ de l'impôt sur le revenu des contribuables selon les Länder et est prélevé par l'employeur, l'administration fiscale le redistribuant ensuite aux Églises et religions concernées moyennant le prélèvement d'environ 3\% à 5\% des sommes recueillies. Un impôt d'Église forfaitaire (Kirchenbeitrag) a par ailleurs été créé dans certains Länder à destination des membres des collectivités de droit public qui ne sont pas soumis à l'impôt sur le revenu. Au Danemark, l'impôt d'Église qui représente $90 \%$ du budget de l'Église luthérienne est collecté par les municipalités et versé par les seuls membres de cette Église nationale, qui ont la faculté de la quitter. La perception de l'impôt cultuel (Kirchenbeitrag) en Autriche (78\% du budget des Églises) relève directement des sociétés religieuses légalement reconnues par l'État et constituées en collectivités de

9. La laicité organisée est reconnue en Belgique par la loi du 21 juin 2002 relative au Conseil central des communautés philosophiques non confessionnelles de Belgique, aux délégués et aux établissements chargés de la gestion des intérêts matériels et financiers des communautés philosophiques non confessionnelles reconnues. 
droit public. Actuellement, la collecte de cet impôt qui correspond à 1,1\% des revenus imposables est gérée par l'Église catholique, l'Église protestante et l'Église vieille catholique conformément à une loi de 1939. Une procédure de sortie des sociétés religieuses reconnues par l'État est prévue par le droit autrichien.

L'impôt d'Église constitue en règle générale la part la plus significative (60 à 80\%) des revenus des confessions religieuses qui bénéficient de ce mécanisme ${ }^{10}$. Il est complété par des dotations de l'État versées en compensation de la sécularisation des biens ecclésiastiques et destinées en principe à la rémunération du clergé : $12 \%$ versés par l'État danois et 12\% par le ministère des Affaires religieuses à l'Église nationale du Danemark, $8 \%$ environ du total de leurs revenus versés par les Länder aux Églises territoriales protestantes et aux diocèses catholiques en Allemagne ${ }^{11}$ et une dotation annuelle versée à la seule Église catholique en Autriche.

En Italie, le système du bénéfice attaché à un office ecclésiastique a disparu en 1984 conformément aux accords de Villa Madame. Les revenus de ces bénéfices étaient auparavant complétés par l'État qui versait la différence - la portion congrue - lorsqu'ils n'atteignaient pas un plafond minimum fixé par les textes. Cette portion congrue à la charge de l'État a alors été remplacée par la possibilité pour chaque contribuable d'assigner $0,8 \%$ de son impôt sur le revenu à l'Église catholique, à une des confessions religieuses qui ont conclu un accord avec l'État ou à l'État italien pour des fins à caractère social. La part correspondant aux contribuables n'ayant pas opté pour l'assignation est répartie entre les différents bénéficiaires proportionnellement au choix des contribuables qui se sont prononcés. Ainsi, $81 \%$ des sommes non affectées ont été reversés à l'Église catholique en 2010. Les autres confessions religieuses conventionnées perçoivent chacune environ $4 \%$ des sommes non affectées par les contribuables ${ }^{12}$. Il en va différemment pour l'Espagne où seule l'Église catholique jouit d'un mécanisme similaire. Chaque contribuable peut assigner $0,7 \%$ de la totalité de ses impôts sur le revenu (part nationale et part des

10. Pulte M., Hense A., Grund und Grenzen staatlicher Religionsförderung unter besonderer Berücksichtigung des Verhältnisses von Staat und Katholischer Kirche in Deutschland, Paderborn, Schöningh Paderborn, 2014.

11. Convention du 23 juin 1960 entre le Saint-Siège et la République d'Autriche portant réglementation des relations patrimoniales.

12. Pacillo O., «Public funding of religious groups in Italy », in Messner, F., Public funding of religions in Europe, Farnham, Ashgate, 2015, p. 149-169. - AstORRI R., « Le financement des confessions religieuses en Italie », in BASDEvant-Gaudemet B. and BerLingò S. (eds.), The financing of religious communities in the European Union. Proceedings of the conference, Messina, 16-19 Nov. 2006, Leuven, Peeters, 2009, p. 223-234. 
régions autonomes) à l'Église catholique ou à des organisations non gouvernementales ${ }^{13}$. Il s'agit, à l'instar de l'Italie, d'une subvention publique versée à une confession religieuse suite à une décision prise par le contribuable, et non d'un impôt spécifique versé par les seuls membres d'une religion. Seuls les contribuables qui en font le choix financent l'Église catholique, la part de ceux qui s'abstiennent est créditée à des ONG poursuivant des fins sociales. Une dotation est versée par l'État sous forme d'avances mensuelles dont le montant est déterminé dans la loi de finances annuelle. Une régularisation intervient dès que l'impôt perçu a été calculé par l'administration fiscale ${ }^{14}$.

\subsection{UNE BOITEUSE INTERDICTION DE FINANCER}

Une troisième catégorie de modes de financement se caractérise par l'interdiction ou la suppression de tout financement public des cultes, sur fond de tensions ou de manière sereine et négociée. L'interdiction peut être inscrite dans la Constitution (Irlande) ou dans la loi (France), et le subventionnement est parfois inexistant sans qu'il soit pour autant prohibé par les textes (Pays-Bas). En Irlande, l'interdiction du financement est posée par l'article 44.2 de la Constitution du $1^{\text {er }}$ juillet 1937 qui dispose que l'État s'engage à ne subventionner aucune religion. Les Églises et religions irlandaises ne recevaient aucun soutien financier des pouvoirs publics lorsque l'Irlande faisait partie du Royaume-Uni et la Constitution irlandaise s'inscrit dans cette continuité. Contrairement à la France où les débats ont été vifs (loi du 9 décembre 1905), les Pays-Bas sont tardivement et paisiblement sortis du système de compensation lors de la révision constitutionnelle de 1972. Cette modification du texte constitutionnel a mis fin en 1983 aux obligations de l'État qui était tenu jusqu'alors de rémunérer les ministres du culte protestants et catholiques. En contrepartie, les pouvoirs publics ont versé une dotation aux Églises concernées pour solde de tout compte ${ }^{15}$. En régime français de séparation,

13. Accord du 3 janvier 1979 entre l'État espagnol et le Saint-Siège sur les questions économiques.

14. L'État espagnol a par ailleurs mis en place un subventionnement des confessions religieuses par le biais de la fondation Pluralismo y convivencia. Cet essai significatif est louable, mais n'a cependant pas atteint son objectif qui est de favoriser « le vivre ensemble dans une société pluraliste». Les subventions sont essentiellement réparties entre les fédérations rassemblant les communautés protestantes, juives et musulmanes qui ont conclu un accord avec le gouvernement espagnol. Il s'agit par ce biais de compenser la différence de traitement entre ces trois groupements et l'Église catholique subventionnée par l'assignation d'une partie de l'impôt sur le revenu.

15. Bisterfeld S. C. van, «State and Church in the Netherlands », in Robbers G. (ed.), op. cit., p. $367-390$. 
l'article 2 de la loi du 9 décembre 1905 dispose que «La République ne reconnaît, ne salarie ni ne subventionne aucun culte ». Le financement direct des cultes n'est cependant pas inexistant dans ces trois pays. En Irlande, les aumôniers et les professeurs de religion sont payés par l'État. En France, les aumôniers de l'armée, des hôpitaux et des prisons sont également rémunérés par l'administration, et les communes peuvent financer la conservation des édifices du culte dont elles ont la propriété et les travaux de réparation de ceux appartenant aux associations cultuelles. Enfin, en l'absence de textes prohibant un soutien économique des confessions religieuses, les pouvoirs locaux néerlandais subventionnent la construction d'édifices cultuels des religions minoritaires en vue de favoriser l'égalité en matière religieuse.

\section{DE LA CRITIQUE DES SYSTÈMES EXISTANTS À DE NOUVEAUX MODES DE FINANCEMENT}

Les normes encadrant le financement des cultes ne sont pas toujours en concordance avec le contexte socioreligieux actuel (2.1) et peinent à instaurer une utopique égalité de traitement (2.2), alors que de nouvelles politiques de subventionnement tendent à instaurer des pratiques en adéquation avec les évolutions sociales (2.3).

\subsection{UN HÉRITAGE NORMATIF EN DÉCALAGE AVEC LES RÉALITÉS SOCIALES, POLITIQUES ET RELIGIEUSES}

Les modes de financement des cultes par les pouvoirs publics, ou avec leur soutien, sont fortement marqués par l'histoire des différents États européens. Dans les entretiens sociologiques menés dans le cadre du projet européen de recherche Religare ${ }^{16}$, tout comme dans un rapport sur la réforme de la législation sur les cultes et les organisations philosophiques non confessionnelles en Belgique d'octobre 2010, il est encore fait mention de la théorie de la compensation pour justifier le financement public des cultes, théorie qui n'a plus qu'une résonance limitée aujourd'hui. Les arguments juridiques et sociopolitiques traditionnellement invoqués sont dorénavant inaudibles par une grande partie de la population et des acteurs sociaux et ils ont été fort logiquement remplacés par des justifications plus adaptées. S'il est possible

16. Projet de recherche RELIGARE - Religious diversity and secular models in Europe financé par le $7^{\mathrm{e}}$ programme-cadre de la Commission européenne (2010-2013). 
de modifier un argumentaire, il est cependant plus difficile de modifier un arsenal juridique datant du début du $\mathrm{XIX}^{\mathrm{e}}$ siècle pour le mettre en adéquation avec l'organisation actuelle des cultes et les impératifs des collectivités publiques touchées par la crise financière. La sécularisation de la société, une hiérarchie des priorités communales différente de celle qui prévalait à l'époque, la réduction actuelle des budgets publics et la pluralisation religieuse ont généré de nouvelles postures des pouvoirs publics dans leurs rapports avec les cultes. Au Luxembourg par exemple, l'article 106 de la Constitution garantissant la rémunération des ministres du culte par l'État devrait être remplacé par un nouvel article qui ne fait plus mention de cette obligation ${ }^{17}$. Conformément aux conventions conclues avec l'État en janvier 2015, les cultes catholique, anglican, protestant, orthodoxe, juif et musulman acceptent en effet que leurs collaborateurs, engagés désormais sous un régime de droit privé, ne soient plus rémunérés sur le budget de l'État, en échange d'une dotation versée par ce dernier. Cette solution semble être acceptée par $61 \%$ de la population luxembourgeoise ${ }^{18}$ et n'a pas suscité de développements doctrinaux sur le statut de ces personnels. En Allemagne, où la Constitution fixe le statut et les modalités de financement des sociétés religieuses et de l'entretien des prêtres par l'impôt d'Église, les évêques catholiques et les chanoines des chapitres cathédraux continuent d'être logés et rémunérés par certains Länder, en contrepartie de la sécularisation de leurs biens en 1803. Les dispositions concordataires qui prévoient cette obligation sont considérées par les évêques eux-mêmes comme inadaptées au contexte actuel. Ils souhaitent la suppression de ce reliquat de l'histoire ${ }^{19}$. Au Danemark, la Cour constitutionnelle a justifié le subventionnement des traitements des pasteurs en s'appuyant sur leur participation au bon fonctionnement d'un service public, en raison de leur implication dans la tenue des registres d'état civil et la gestion des cimetières. Le statut de fonctionnaire des pasteurs de l'Église nationale danoise découle de leur mission de participation à un service public de l'État et non de la qualification des activités de cette Église de service public ${ }^{20}$.

17. «En matière religieuse et idéologique, l'État respecte, en vertu du principe de séparation, les principes de neutralité et d'impartialité. La loi règle les relations entre l'État et les communautés religieuses ainsi que leur reconnaissance. Dans les limites et formes fixées par la loi, des conventions à approuver par la Chambre des Députés peuvent préciser les relations entre l'État et les communautés religieuses reconnues".

18. Sondage TNS ILRES PolitMonitor, février 2015.

19. Ramelsberger A., «Katholiken wollen Bischöfe selbst bezahlen », Süddeutsche Zeitung, 4 August 2010.

20. Christoffersen L., «A long historical path towards transparency, accountability and good governance: on financing religions in Denmark », in Messner, F., Public funding of religions in Europe, op. ,cit., p. 125-147. 


\subsection{LE DIFFICILE NIVELAGE DES DIFFÉRENCES DE TRAITEMENT}

Ces systèmes de financement des cultes marqués par l'empreinte de l'histoire des États et des religions de la « grande majorité de la population » ont fort logiquement engendré des traitements différenciés entre religions, et plus récemment entre religions et familles de pensée (athées, humanistes). Les tentatives de résorption de ces différences de traitement, par le biais de l'élargissement des avantages financiers aux traditions religieuses ou groupements philosophiques non confessionnels les plus significatifs, n'ont pas corrigé tous les écarts et parfois même créé de nouvelles disparités. En Italie par exemple, l'Église catholique dont les relations avec l'État sont fixées par un accord concordataire bénéficie non seulement d'un traitement privilégié à l'occasion de la répartition de "l'impôt assigné ", mais également de la prise en charge par l'administration de la rémunération des aumôniers et des enseignants de religion des écoles, collèges et lycées publics. Les confessions religieuses qui ont conclu avec l'État des accords ratifiés par le Parlement perçoivent l'impôt assigné, mais les coûts liés à l'organisation de l'enseignement religieux de ces confessions et la rémunération de leurs aumôniers ne sont pas pris en charge par l'administration. La doctrine suggère par ailleurs que toutes les religions, conventionnées ou non, devraient être éligibles au mécanisme de l'affectation de l'impôt ${ }^{21}$ et les sommes non assignées pourraient être reversées à l'État et non à la religion majoritaire. Les autres confessions religieuses, non conventionnées, sont organisées dans le cadre d'une loi de 1929 qui leur confère la personnalité juridique, mais ne prévoit aucun financement direct. Dans le système espagnol d'assignation de l'impôt, seule l'Église catholique bénéficie de ce mécanisme. Les trois fédérations de confessions religieuses conventionnées (protestants, musulmans, juifs) sont quant à elles exonérées d'un certain nombre de taxes et d'impôts, alors que les confessions enregistrées sont exclues à la fois de l'assignation et des exonérations fiscales. En Belgique, six religions (protestants, catholiques, orthodoxes, juifs, musulmans, anglicans) et une fédération d'associations humanistes, également dénommée « laïcité organisée » ou « groupements philosophiques non confessionnels », ont été reconnues par la loi et sont par voie de conséquence financées par l'État, les régions et les communes. Mais en dépit de ces efforts, le régime belge des cultes reconnus continue d'être sous le feu de la critique en raison de la disparité qu'il instaure entre les six groupements reconnus, la laïcité organisée et les cultes non reconnus. 
Adossée au seul argument d'une égalité par ailleurs inatteignable, la démultiplication des reconnaissances et des conventionnements engendre de nouvelles frustrations. Par conséquent, le système actuel du financement public des cultes est souvent perçu comme injuste par une part croissante d'une population constituée d'indifférents et d'athées, de membres de minorités religieuses, mais également de membres des religions "privilégiées », certes attachés aux diverses spécificités nationales, mais qui sont embarrassés par des modalités de soutien qui paraissent disproportionnées. Ces exemples soulignent l'existence de mécanismes de financement dont la complexité s'accroît à l'occasion des tentatives de résorption de différences de traitement. Les religions culturellement majoritaires ou historiques, qui ont sur une longue période contribué et contribuent encore au façonnage de l'identité nationale, continuent malgré certains efforts à bénéficier d'avantages spécifiques.

\subsection{UN FINANCEMENT ATTRIBUÉ EN CONTREPARTIE D'UNE CONTRIBUTION AU VIVRE ENSEMBLE ET AU MAINTIEN DU CONSENSUS SOCIAL}

Les modèles historiques de financement des confessions religieuses ne constituent pas des particularités indépassables. Ainsi, nombre d'anciens États communistes ont à l'occasion de la redéfinition des relations entre l'État et les religions mis en place des mécanismes banalisés de soutien économique aux institutions religieuses, sous forme de subventions relevant du droit commun et d'appels à projets. L'Estonie, qui finance les activités sociales et culturelles de diverses confessions religieuses, verse une subvention modeste au Conseil des Églises estoniennes qui l'utilise librement. La Lituanie soutient les seules activités culturelles, alors que la Slovénie a mis en place un système de financement sur projets. Ni le concordat entre la République slovène et le Saint-Siège, ni la loi slovène sur la liberté religieuse ne prévoient de financement des cultes et des exonérations fiscales sont accordées en raison de l'importance du rôle social joué par les religions. Les confessions religieuses bénéficiaires doivent mener des activités à caractère culturel. La législation slovaque prévoit quant à elle la possibilité pour les contribuables d'affecter 1\% de l'impôt sur le revenu à un groupement d'intérêt général de leur choix ; les religions enregistrées en font partie. L'objectif des pouvoirs publics est dans ces quelques exemples clairement affiché : le subventionnement des confessions religieuses est aligné sur celui des associations prônant un engagement au service de toute la collectivité, ou du moins utile à la société. 
Cette tendance se développe également dans les États dont les statuts des cultes ont été façonnés par et pour les cultes historiques. Ainsi en Allemagne, l'accord passé en 2012 entre la ville-État de Hambourg et la communauté des alévis, ainsi que celui conclu la même année avec la communauté musulmane turque, insistent sur l'engagement de celles-ci en faveur de la tolérance envers les autres religions et conceptions de l'univers, et sur leur respect des positions doctrinales hétérodoxes au sein de leur propre confession (art. 1 des accords). Elles doivent adhérer à un socle de valeurs communes, respecter les droits fondamentaux et la démocratie, s'opposer à la discrimination en raison de l'origine, des convictions, du sexe et de l'orientation sexuelle des personnes (art. 2). Elles sont tenues d'exprimer leur volonté d'intégration dans la société en participant à des activités avec d'autres groupements intermédiaires, en valorisant la dimension culturelle de leur patrimoine religieux et en collaborant avec les pouvoirs publics sur des sujets d'intérêt commun (préambule). La ville-État de Hambourg et les communautés alévie et musulmane s'engagent en outre de concert à promouvoir l'égalité entre les hommes et les femmes dans la société (art. 2). En contrepartie, la ville-État subventionne un cours d'enseignement religieux musulman dans les établissements d'enseignement publics et finance la formation des maîtres de religion à l'Université de Hambourg (art. 5). Si elle s'engage par ailleurs à faciliter la création de cimetières publics et privés musulmans, la construction de mosquées, la création d'écoles, de collèges et de lycées privés musulmans, l'accès aux médias et aux aumôneries et la reconnaissance de jours fériés musulmans au sens de la loi de Hambourg sur les jours fériés, il faut noter que les seuls financements explicitement prévus par les accords s'appliquent à l'enseignement et à la formation des maîtres, dont les contenus doivent recevoir l'aval des pouvoirs publics. Ces accords permettent d'intégrer la transmission religieuse dans la méthodologie de l'enseignement public tout en favorisant la connaissance des autres religions. Seul le culte musulman fait jusqu'à présent l'objet d'une telle procédure en Allemagne.

Le financement de la formation des cadres religieux est une illustration de la volonté des pouvoirs publics de promouvoir un subventionnement utile à la société. Représentants de l'autorité religieuse dans leur communauté, les cadres religieux jouent un rôle important dans l'intégration des ressortissants de pays tiers et sont également en contact avec les responsables et les membres d'autres communautés religieuses et d'autres groupements intermédiaires. Une attention toute particulière est portée aux religions nouvellement implantées avec l'objectif de les intégrer dans la société, soit par le biais d'une éducation théologique dans les universités publiques ou encore grâce à la création de 
diplômes spécifiques de formation civile et civique. Les États dont les textes prévoient la création de facultés de théologie dans les universités publiques, ou privées accréditées par l'État, sont moins concernés par la mise en place de formations civiles et civiques des cadres religieux. Il s'agit essentiellement de l'Allemagne, de l'Autriche, du Royaume-Uni, de la Suède et de la Grèce ou les facultés de théologie font partie des composantes de l'université publique. La récente loi autrichienne sur l'islam du 12 avril 2015 prévoit la création de toutes pièces d'une faculté de théologie musulmane à Vienne en 2016, les sociétés religieuses musulmanes reconnues renonçant en contrepartie au financement, notamment de la rémunération des imams, par les États étrangers. En Allemagne, le ministère fédéral de l'Enseignement supérieur s'est engagé à financer pendant cinq ans des postes de professeurs dans les instituts de théologie et de pédagogie religieuse islamique ${ }^{22}$. Les universités de Tübingen, Münster, Osnabrück, Francfort-sur-le-Main et Giessen bénéficient de ce soutien spécifique depuis 2011. Le Conseil des sciences (Wissenschaftsrat) qui conseille l'État fédéral et les États fédérés pour toutes les questions relatives à la recherche et à l'enseignement supérieur, estime que l'établissement de facultés de théologie musulmane dans les universités publiques garantit la qualité de l'enseignement et de la recherche, permet la confrontation avec d'autres formes de pensée et fournit les bases du dialogue interreligieux ${ }^{23}$.

\section{CONCLUSION}

L'évolution des modes de financement des cultes en Europe reflète la place et le rôle octroyés par les autorités publiques aux Églises et religions dans la société et au sein de l'État. Alors que le Moyen-Âge voit un enchevêtrement des pouvoirs spirituels et temporels, il apparaît que dès le XVI ${ }^{\mathrm{e}}$ siècle la sécularisation des biens ecclésiastiques tend moins à enrichir les princes qu'à conforter leur pouvoir en réduisant la puissance économique de l'Église. Les tentatives de reconstitution d'un patrimoine garantissant une ample autonomie de l'Église au cours des $\mathrm{XIX}^{\mathrm{e}}$ et $\mathrm{XX}^{\mathrm{e}}$ siècle se sont fort logiquement soldées par des échecs et les systèmes des cultes instaurés au XIX ${ }^{e}$ siècle ont en règle générale mal vieilli.

22. WisSENSCHAFTSRAT, Empfehlungen zur Weiterentwicklung von Theologien und religionsbezogenen Wissenschaften an deutschen Hochschulen, 29 janv. 2010.

23. V. MESSNER F., La formation des cadres religieux musulmans, rapport remis à la ministre de l'Éducation nationale, de l'Enseignement supérieur et de la Recherche et au ministre de l'Intérieur, 2015. 
L'impôt d'Église collecté aujourd'hui en Allemagne par l'administration fiscale a certes été contesté en raison de la publicité donnée à l'affiliation ou à la non-affiliation religieuse du contribuable, mais il relève du volontariat : seuls les membres d'une société religieuse constituée en collectivité de droit public versent leur quote-part. Par contre, le manque de transparence et de démocratie dans la gestion des biens appartenant à l'Église catholique a été sévèrement critiqué et a contribué à un nombre élevé de sorties d'Église dans les diocèses concernés. La teneur des arguments propres à justifier le financement public des cultes s'est affinée ces dernières années, notamment grâce à la jurisprudence et à la doctrine juridique. Le législateur et le juge ont essentiellement retenu l'intérêt collectif ${ }^{24}$ pour justifier les soutiens économiques apportés aux institutions cultuelles. Les activités cultuelles ont un caractère d'intérêt général en ce qu'elles contribuent à la constitution du lien social et les institutions religieuses développent des activités socio-caritatives à destination de l'ensemble de la collectivité. Les évolutions les plus récentes sont encore plus significatives. Le financement public est subordonné à un engagement des autorités cultuelles d'adhérer au socle des valeurs communes et de favoriser l'intégration des communautés religieuses dans la société. Considérées pendant des siècles comme des services publics sui generis, les religions sont actuellement dans nombre d'États européens soutenues par les pouvoirs publics pour leur capacité à créer du lien social et de l'intégration et bénéficient à ce titre des avantages accordés aux associations d'intérêt général.

24. V. dans ce numéro, Prélot P.-H., « Le financement public des cultes en France entre régime d'exception et droit commun ». 